\title{
Chiral Symmetry Restoration and Pion Properties in a $q$-Deformed NJL Model
}

\author{
V. S. Timóteo \\ Centro Superior de Educação Tecnológica, Universidade Estadual de Campinas, 13484-370, Limeira, SP, Brazil \\ and C. L. Lima \\ Instituto de Física, Universidade de São Paulo, CP 66318, 05315-970, São Paulo, SP, Brazil
}

Received on 27 October, 2005

\begin{abstract}
We review the implementation of a $q$-deformed fermionic algebra in the Nambu-Jona-Lasinio model (NJL). The gap equations obtained from a deformed condensate as well as from the deformation of the NJL Hamiltonian are discussed. The effect of both temperature and deformation in the chiral symmetry restoration process as well as in the pion properties is studied.
\end{abstract}

Keywords: Deformed algebras; Effective models; Chiral symmetry

\section{INTRODUCTION}

Chiral symmetry breaking is an important phenomena in hadron physics and is of fundamental importance for hadron properties. The difficulties involved in obtaining low-energy properties directly from $\mathrm{QCD}$, the fundamental theory of strong interactions, have motivated the construction of effective models. Due to its simplicity and effectiveness in describing hadrons at low energies, the NJL model [1] has become the most familiar effective model for strong interactions.

The standard scenario for spontaneous chiral symmetry breaking is the arising of a quark condensate. In the NJL model the condensates appear when the strength of the contact interaction exceeds a critical value, separating the WignerWeyl and Nambu-Goldstone realizations of chiral symmetry. The condensate $<\bar{q} q>$ is the order parameter.

Apart from giving a reasonable description of the light mesons, the NJL model is also very useful for studying the chiral symmetry breaking process as well as its restoration at finite temperature $[2,3]$. The NJL model is in addition very suitable for testing new ideas. In previous works, we have investigated the effect of $q$-deformation in the chiral symmetry breaking process within the context of the NJL model. In particular, we observed how the condensate is affected by the deformation. As a direct consequence, the dynamical quark mass, the chiral symmetry breaking and restoration processes are accordingly affected $[4,5]$.

The aim of this work is to study the effect of both temperature and deformation in the chiral symmetry restoration, and evaluate the pion properties in a $q$-deformed NJL model at finite temperature. This paper is organized as follows. In Sec. II briefly review the Nambu-Jona-Lasinio model, Sec. III introduces the $q$-deformed fermionic algebra which will be used along this work, and shows how the deformed algebra is implemented in the NJL model. In Sec. IV, we present the investigation of the chiral symmetry restoration with both finite temperature and $q$-deformation. Finally, Sec. V contains our main conclusions.

\section{THE NAMBU-JONA-LASINIO MODEL}

The Nambu-Jona-Lasinio model was first introduced to describe the nucleon-nucleon interaction via a four-fermion contact interaction. Later, the model was extended to quark degrees of freedom becoming an effective model for quantum chromodynamics.

The Lagrangian of the NJL model is given by

$$
\begin{gathered}
\mathcal{L}_{N J L}=\bar{\psi} i \gamma^{\mu} \partial_{\mu} \psi+\mathcal{L}_{i n t}, \\
\mathcal{L}_{i n t}=G\left[(\bar{\psi} \psi)^{2}+\left(\bar{\psi} i \gamma_{5} \tau \psi\right)^{2}\right] .
\end{gathered}
$$

Linearizing the above interaction in a mean field approach, the last term does not contribute if the vacuum is parity and Lorentz invariant. The Lagrangian with the linearized interaction is then

$$
\mathcal{L}_{N J L}=\bar{\psi} i \gamma^{\mu} \partial_{\mu} \psi+2 G\langle\bar{\psi} \psi\rangle \bar{\psi} \psi
$$

Regarding this Lagrangian as a Dirac Lagrangian for massive quarks we obtain a dynamical mass for the quarks

$$
m=-2 G\langle\bar{\psi} \psi\rangle
$$

where $\langle\bar{\psi} \psi\rangle$ is the vacuum expectation value of the scalar density $\bar{\psi} \psi$, representing the quark condensates. Eq. (4) describes how the dynamical mass is generated with the appearance of the quark condensates. The quarks are massless if the condensate vanishes.

From the anti-commutation relations for the fermionic fields, the condensate can be written as

$$
\langle\bar{\psi}(x) \psi(x)\rangle=-\operatorname{Tr} \lim _{y \rightarrow x+}\langle T[\psi(x) \bar{\psi}(y)]\rangle,
$$

where

$$
\langle T[\psi(x) \bar{\psi}(y)]\rangle=i S_{F}(x-y),
$$

and $S_{F}(x-y)$ is the Feynman propagator in the configuration space. In the momentum space this propagator is given by

$$
S_{F}(p)=\frac{\gamma^{\mu} p_{\mu}+m}{p^{2}-m^{2}+i \varepsilon} .
$$


A Fourier transformation let us write the condensate in the following fashion

$$
\langle\bar{\psi}(x) \psi(x)\rangle=-i \lim _{y \rightarrow x+} \operatorname{Tr} \int \frac{d^{4} p}{(2 \pi)^{4}} S_{F}(p) e^{-i(x-y) p},
$$

which after a 3D non-covariant cutoff becomes

$$
\langle\bar{\psi} \psi\rangle=-\frac{N_{c} N_{f}}{\pi^{2}} \int_{0}^{\Lambda} d p p^{2} \frac{m}{\sqrt{\mathbf{p}^{2}+m^{2}}},
$$

where $\phi(p)=2 \theta(p), N_{c}$ is the number of colors and $N_{f}$ is the number of flavors. Using this result we obtain the NJL gap equation in its more familiar form (see reference [3])

$$
m=2 N_{c} N_{f} \frac{1}{\pi^{2}} \int_{0}^{\Lambda} d p p^{2} \frac{m}{\sqrt{\mathbf{p}^{2}+m^{2}}} .
$$

\section{A. The Bogoliubov-Valatin approach}

The Bogoliubov-Valatin variational approach is an alternative way to obtain the NJL gap equation and observe the dynamical chiral symmetry breaking process [6]. The variational approach to obtain the gap equation consists on the following procedure: a) to define a variational vacuum, b) to calculate the vacuum expectation value of the Hamiltonian, obtaining the functional for the total energy, and c) to minimize the functional, obtaining the variational parameters and the gap equation. For this purpose we start with the NJL Hamiltonian corresponding to the Lagrangian Eq.(1)

$$
H_{N J L}=-i \bar{\psi} \gamma \cdot \nabla \psi-G\left[(\bar{\psi} \psi)^{2}+\left(\bar{\psi} i \gamma_{5} \tau \psi\right)^{2}\right]
$$

and make a Fourier decomposition in the quark fields

$\psi(x, 0)=\sum_{s} \int \frac{d^{3} p}{(2 \pi)^{3}}\left[b(\mathbf{p}, s) u(\mathbf{p}, s) e^{i \mathbf{p} \cdot \mathbf{x}}+d^{\dagger}(\mathbf{p}, s) v(\mathbf{p}, s) e^{-i \mathbf{p} \cdot \mathbf{x}}\right]$,

defining the annihilation operators $b(\mathbf{p}, s)$ and $d(\mathbf{p}, s)$ of particles and anti-particles with momentum $\mathbf{p}$ and helicity $s$, which destroy the bare vacuum $|0\rangle$ :

$$
b(\mathbf{p}, s)|0\rangle=d(\mathbf{p}, s)|0\rangle=0 .
$$

The spinors $u(\mathbf{p}, s)$ and $v(\mathbf{p}, s)$ are normalized helicity eigenstates satisfying

$$
\begin{gathered}
\gamma_{\mu} p^{\mu} u(\mathbf{p}, s)=\gamma_{\mu} p^{\mu} v(\mathbf{p}, s)=0, \\
u^{\dagger}(\mathbf{p}, s) u(\mathbf{p}, s)=v^{\dagger}(\mathbf{p}, s) v(\mathbf{p}, s)=1 .
\end{gathered}
$$

We now introduce the following BCS-like vacuum

$$
|N J L\rangle=\prod_{\mathbf{p}, s= \pm 1}\left[\cos \theta(p)+s \sin \theta(p) b^{\dagger}(\mathbf{p}, s) d^{\dagger}(-\mathbf{p}, s)\right]|0\rangle
$$

which, for a given momentum $\mathbf{p}$, is expanded as

$$
\begin{aligned}
|N J L\rangle & =\cos ^{2} \theta(p)|0\rangle \\
& +\sin \theta(p) \cos \theta(p) b^{\dagger}(\mathbf{p},+) d^{\dagger}(-\mathbf{p},+)|0\rangle \\
& -\sin \theta(p) \cos \theta(p) b^{\dagger}(\mathbf{p},-) d^{\dagger}(-\mathbf{p},-)|0\rangle \\
& -\sin ^{2} \theta(p) b^{\dagger}(\mathbf{p},-) d^{\dagger}(-\mathbf{p},-) b^{\dagger}(\mathbf{p},+) d^{\dagger}(-\mathbf{p},+)|0\rangle .
\end{aligned}
$$

The operators which annihilate this vacuum are given by the Bogoliubov-Valatin transformations

$$
\begin{gathered}
B(\mathbf{p}, s)=\cos \theta(p) b(\mathbf{p}, s)+s \sin \theta(p) d^{\dagger}(-\mathbf{p}, s), \\
D(\mathbf{p}, s)=\cos \theta(p) d(\mathbf{p}, s)-s \sin \theta(p) b^{\dagger}(-\mathbf{p}, s), \\
B(\mathbf{p}, s)|N J L\rangle=D(\mathbf{p}, s)|N J L\rangle=0 .
\end{gathered}
$$

To calculate the expectation value of the NJL Hamiltonian with respect to the state Eq. (15)

$$
\left\langle N J L\left|\mathcal{H}_{N J L}\right| N J L\right\rangle
$$

it is convenient to express $\psi$ in terms of the quasi-particles operators $B(\mathbf{p}, s)$ and $D(\mathbf{p}, s)$ as

$$
\psi(x, 0)=\sum_{s} \int \frac{d^{3} p}{(2 \pi)^{3}}\left[B(\mathbf{p}, s) M_{1}(\mathbf{p}, s) e^{i \mathbf{p} \cdot \mathbf{x}}+D^{\dagger}(\mathbf{p}, s) M_{2}(\mathbf{p}, s) e^{-i \mathbf{p} \cdot \mathbf{x}}\right]
$$

where

$$
\begin{aligned}
& M_{1}(\mathbf{p}, s)=\left[\cos \theta(p)+\gamma^{0} \sin \theta(p)\right] u(\mathbf{p}, s), \\
& M_{2}(\mathbf{p}, s)=\left[\cos \theta(p)-\gamma^{0} \sin \theta(p)\right] u(\mathbf{p}, s) .
\end{aligned}
$$

Making use of the spinor relations

$$
\gamma_{0} s v(-\mathbf{p}, s)=u(\mathbf{p}, s),
$$




$$
\sum_{s} u(\mathbf{p}, s) u^{\dagger}(\mathbf{p}, s)=\frac{1}{2}\left(1-\gamma \cdot \frac{\mathbf{p}}{|\mathbf{p}|} \gamma^{0}\right),
$$

the functional for the total energy in the Hartree approximation is obtained as

$$
W[\phi]=-2 N_{c} N_{f} \int \frac{d^{3} p}{(2 \pi)^{3}} p \cos \phi(p)-4 G\left(N_{c} N_{f}\right)^{2}\left[\int \frac{d^{3} p}{(2 \pi)^{3}} \sin \phi(p)\right]^{2} .
$$

The form of $\phi(p)$ which minimizes the total energy Eq. (26) is obtained from the condition

$$
\frac{\delta W[\phi]}{\delta \phi(p)}=0 .
$$

This procedure yields to

$$
p \tan \phi(p)=4 G N_{c} N_{f} \int \frac{d^{3} p^{\prime}}{(4 \pi)^{3}} \sin \phi\left(p^{\prime}\right)
$$

which becomes

$$
m=4 G N_{c} N_{f} \int \frac{d^{3} p}{(2 \pi)^{3}} \frac{m}{\sqrt{\mathbf{p}^{2}+m^{2}}},
$$

provided the variational angles acquire the following form

$$
\tan \phi(p)=\frac{m}{p}, \quad \sin \phi(p)=\frac{m}{\sqrt{\mathbf{p}^{2}+m^{2}}} .
$$

\section{III. $q$-DEFORMATION IN THE NJL MODEL}

In this section we discuss the implementation of the deformed algebra to the NJL model. The $q$-deformed fermionic algebra [7] that we shall use is based in the work of Ubriaco [8], where the thermodynamic properties of a many fermion system were studied. In the construction of a $q$-covariant form of the BCS approximation [9], it was shown that the creation and annihilation operators of the $s u_{q}(2 j+1)$ fermionic algebra are given by

$$
\mathcal{A}_{j m_{j}}=a_{j m_{j}} \prod_{i=m_{j}+1}\left(1+Q a_{j i}^{\dagger} a_{j i}\right)
$$

$$
\mathcal{A}_{j m_{j}}^{\dagger}=a_{j m_{j}}^{\dagger} \prod_{i=m_{j}+1}\left(1+Q a_{j i}^{\dagger} a_{j i}\right)
$$

where $Q=q^{-1}-1, j=1 / 2$ and $m_{j}= \pm 1 / 2$. As discussed in [4], the first consequence of the above deformation is that only the operators corresponding to $m_{j}=-\frac{1}{2}$ are modified, meaning that only negative helicity quarks (anti-quarks) operators will be deformed since in the NJL model we deal with quarks (anti-quarks) creation and annihilation operators.

We have two different approaches to obtain a new gap equation. The first one consists in to perform the $q$-deformation in the condensate, which is a part of the self-consistent equation for the dynamical mass. In the second one the $q$ deformation is performed in the NJL Hamiltonian, and we use the Bogoliubov-Valatin procedure to obtain the new gap equation. We now turn to a detailed discussion of these two different approaches.

\section{A. Deforming the condensates}

To obtain the deformed gap equation we work with the BCS-like vacuum (15) in the standard Bogoliubov-Valatin variational approach. The quark fields are expressed in terms of $q$-deformed creation and annihilation operators as

$$
\psi_{q}(x, 0)=\sum_{s} \int \frac{d^{3} p}{(2 \pi)^{3}}\left[\mathcal{B}(\mathbf{p}, s) u(\mathbf{p}, s) e^{i \mathbf{p} \cdot \mathbf{x}}+\mathcal{D}^{\dagger}(\mathbf{p}, s) v(\mathbf{p}, s) e^{-i \mathbf{p} \cdot \mathbf{x}}\right]
$$

where the $q$-deformed quark and anti-quark creation and annihilation operators $\mathcal{B}, \mathcal{B}^{\dagger}, \mathcal{D}$, and $\mathcal{D}^{\dagger}$, are expressed in terms of the non-deformed ones according to Eqs. (31) and (32)

$$
\begin{array}{ll}
\mathcal{B}_{-}=b_{-}\left(1+Q b_{+}^{\dagger} b_{+}\right) & , \quad \mathcal{B}_{-}^{\dagger}=b_{-}^{\dagger}\left(1+Q b_{+}^{\dagger} b_{+}\right), \\
\mathcal{D}_{-}=d_{-}\left(1+Q d_{+}^{\dagger} d_{+}\right) & , \quad \mathcal{D}_{-}^{\dagger}=d_{-}^{\dagger}\left(1+Q d_{+}^{\dagger} d_{+}\right),
\end{array}
$$




$$
\begin{aligned}
& \mathcal{B}_{+}=b_{+}, \mathcal{B}_{+}^{\dagger}=b_{+}^{\dagger}, \\
& \mathcal{D}_{+}=d_{+}, \mathcal{D}_{+}^{\dagger}=d_{+}^{\dagger},
\end{aligned}
$$

where $+(-)$ stands for the positive (negative) helicity and the notation has been simplified: $(\mathbf{p}, s) \rightarrow s$. We would like to note that, as discussed in Ref. [9], the deformed vacuum differs from the non-deformed one only by a phase and, therefore, the effects of the deformation comes solely from the modified field operators. Additionally, the $q$-deformed NJL Lagrangian, constructed using $\psi_{q}$ instead of $\psi$, is invariant under the quantum group $S U_{q}(2)$ transformations. This can be seen by using the two-dimensional representation of the $S U_{q}(2)$ unitary transformation given in Ref. [8].

The deformed gap equation is

$$
m=-2 G\langle\bar{\psi} \psi\rangle_{q},
$$

where $\langle\bar{\psi} \psi\rangle_{q}$ is the $q$-deformed condensate calculated using the BCS-like vacuum, Eq. (15), and Eq. (11),

$$
\begin{aligned}
\langle\bar{\psi} \psi\rangle_{q} & =\left\langle N J L\left|\bar{\psi}_{q} \psi_{q}\right| N J L\right\rangle \\
& =\langle\bar{\psi} \psi\rangle+\langle N J L|Q| N J L\rangle,
\end{aligned}
$$

where $\langle\bar{\psi} \psi\rangle$ is the non-deformed condensate and $\langle N J L|Q| N J L\rangle$ represents all non-vanishing matrix elements arising from the $q$-deformation of the quark fields. The contribution of these $q$-deformed matrix elements is

$$
\langle N J L|Q| N J L\rangle=Q \int \frac{d^{3} p}{(2 \pi)^{3}}[\sin 2 \theta(p)-\sin 2 \theta(p) \cos 2 \theta(p)] .
$$

The calculation of the deformed condensate will be performed in a similar way as in the usual case. It requires also a regularization procedure since the NJL interaction is not perturbatively renormalizable. For reasons of simplicity a non-covariant trimomentum cutoff is applied arising

$$
\langle\bar{\psi} \psi\rangle_{q}=-\frac{3 m}{\pi^{2}}\left[\left(1-\frac{Q}{2}\right) \int_{0}^{\Lambda} d p \frac{p^{2}}{\sqrt{\mathbf{p}^{2}+m^{2}}}+\frac{Q}{2} \int_{0}^{\Lambda} d p \frac{p^{3}}{\mathbf{p}^{2}+m^{2}}\right]
$$

for each quark flavor. At this point we see that the dynamical mass is again given by a self-consistent equation since the condensate depends also on the mass. Inserting Eq. (41) into Eq. (38) we obtain the deformed NJL gap equation

$$
m=\frac{2 G m}{\pi^{2}}\left[\left(1-\frac{Q}{2}\right) \int_{0}^{\Lambda} d p \frac{p^{2}}{\sqrt{\mathbf{p}^{2}+m^{2}}}+\frac{Q}{2} \int_{0}^{\Lambda} d p \frac{p^{3}}{\mathbf{p}^{2}+m^{2}}\right] .
$$

It is easy to see that for $Q=0(q=1)$, we recover the NJL gap equation in its more familiar form

$$
m=\frac{2 G m}{\pi^{2}} \int_{0}^{\Lambda} d p \frac{p^{2}}{\sqrt{\mathbf{p}^{2}+m^{2}}}+m_{0},
$$

where $m_{0}$ appears only if we consider the current quark mass term $\mathcal{L}_{\text {mass }}=-m_{0} \bar{\psi} \psi$ in the NJL Lagrangian Eq. (1).

The pion decay constant is calculated from the vacuum to one pion axial vector current matrix element, which, in the simple 3D non-covariant cutoff we are using [6], is given by

$$
f_{\pi}^{2}=N_{c} m^{2} \int_{0}^{\Lambda} \frac{d^{3} p}{(2 \pi)^{3}} \frac{1}{\left(\mathbf{p}^{2}+m^{2}\right)^{3 / 2}},
$$

for each quark flavor. The deformed calculation of $f_{\pi}$ is performed directly by substituting the dynamical mass in Eq. (44) from the one obtained in Eq. (42).

As in the non-deformed case, the $q$-gap equation has nontrivial solutions when the coupling $G$ exceeds a critical value
$G_{c}$ related to the cutoff. The dynamical mass is accordingly modified through the deformed gap equation (42).

The behavior of the condensate around the critical coupling, $G_{c}$, is similar for both deformed and non-deformed cases, meaning that the adopted procedure to $q$-deform the underlying $s u(2)$ algebra in a two flavor NJL model does not change the behavior of the phase transition around $G_{c}$. The formalism developed in $[8,9]$ allow $q$-values smaller than one (which corresponds to $Q>0$ ). It is worth to mention that in this case the $q$-deformation effect goes in the opposite direction, namely, the condensate value and the dynamical mass decrease for $q<1$.

\section{B. Deforming the NJL Hamiltonian}

Here it is important to stress again that the deformed version of this vacuum differs from the non-deformed one only by a phase and, therefore, the effects of the deformation comes 
solely from the deformation of the Hamiltonian.

The deformed functional for the total energy will be obtained from the vacuum expectation value of the $q$-deformed NJL Hamiltonian:

$$
\mathcal{W}^{q}[\theta(p)]=\left\langle N J L\left|\mathcal{H}_{N J L}^{q}\right| N J L\right\rangle,
$$

where the $q$-deformed Hamiltonian is

$$
\mathcal{H}_{N J L}^{q}=-i \bar{\psi}_{q} \gamma \cdot \nabla \psi_{q}-G\left(\bar{\psi}_{q} \psi_{q}\right)^{2}-G\left(\bar{\psi}_{q} i \gamma_{5} \psi_{q}\right)^{2},
$$

and $\psi_{q}$ is given by Eq. (33). Due to the additive structure of the $q$-deformation, the deformed Hamiltonian can be written as

$$
\mathcal{H}_{N J L}^{q}=H_{N J L}+\mathcal{H}(Q),
$$

and the functional will have the same form

$$
\mathcal{W}^{q}[\theta(p)]=W[\theta(p)]+\mathcal{W}[Q, \theta(p)] .
$$

The last terms of Eqs. (47) and (48), namely $\mathcal{H}(Q)$ and $\mathcal{W}[Q, \theta(p)]$, stand for the new terms of first order in $Q$ generated when the algebra is deformed, and, therefore, they vanish for $q=1(Q=0)$. The full resulting interaction includes 3-, 4-, 5-, and 6-body interactions, which corresponds to the new terms of order $Q, Q^{2}, Q^{3}$, and $Q^{4}$, respectively. We have considered only the three-body terms of order $Q$.

The non-deformed case, which corresponds to $q=$ $1(Q=0)$, has been discussed in Sec. II. In order to obtain the new gap equation, we need to calculate the new matrix elements arising from the $q$-deformation of the NJL Hamiltonian, and add them to the non-deformed functional. This procedure yields to the full $q$-deformed functional for the total energy:

$$
\begin{aligned}
\mathcal{W}^{q}[\theta(p)]= & -2 N_{c} N_{f}(1+Q) \int \frac{d^{3} p}{(2 \pi)^{3}} p \cos \phi+N_{c} N_{f} \frac{Q}{2} \int \frac{d^{3} p}{(2 \pi)^{3}} p \cos ^{2} \phi \\
& +N_{c} N_{f} \frac{N_{c} N_{f}}{3 \pi^{2}} Q G \Lambda^{3} \int \frac{d^{3} p}{(2 \pi)^{3}} \cos \phi-\left(N_{c} N_{f}\right)^{2} G(4+Q)\left\{\int \frac{d^{3} p}{(2 \pi)^{3}} \sin \phi\right\}^{2} .
\end{aligned}
$$

Defining the new variables

$$
\begin{aligned}
P_{q} & =P+P_{0} \\
P & =(1+Q) p \\
P_{0} & =-\frac{N_{c} N_{f}}{6 \pi^{2}} G \Lambda^{3} Q, \\
K & =-\frac{Q}{2} p \\
G^{\prime} & =G\left(1+\frac{Q}{4}\right),
\end{aligned}
$$

and performing the same minimization procedure as in the non-deformed case we obtain

$$
P_{q} \tan \phi+K \sin \phi=4 G^{\prime} N_{c} N_{f} \int \frac{d^{3} p^{\prime}}{(2 \pi)^{3}} \sin \phi .
$$

The term $K \sin \phi$ was overlooked in a previous paper [5] [14]. Trying to keep as much similarity as possible with the usual solution, instead of solving the equation $P_{q} \tan \phi(p)+$ $K \sin \phi(p)=M$, the $\sin \phi(p)$ will be substituted by $\alpha \tan \phi(p)$, with $\alpha=0.59$ chosen to minimize the difference between $\sin \phi$ and $\tan \phi$ in the interval $[0, \pi / 4]$.

The new gap equation then becomes

$$
M=4 G^{\prime} N_{c} N_{f} \int \frac{d^{3} p}{(2 \pi)^{3}} \frac{M}{\sqrt{\mathbf{K}_{q}^{2}+M^{2}}},
$$

provided the variational angles have the same old structure (but they now are $q$-dependent)

$$
\begin{gathered}
\tan 2 \theta_{q}(p)=\frac{M}{K_{q}}, \sin 2 \theta_{q}(p)=\frac{M}{\sqrt{\mathbf{K}_{q}^{2}+M^{2}}}, \\
\cos 2 \theta_{q}(p)=\frac{K_{q}}{\sqrt{\mathbf{K}_{q}^{2}+M^{2}}},
\end{gathered}
$$

where

$$
K_{q}=P_{q}+\alpha K .
$$

It is easy to see that, when $q \rightarrow 1(Q \rightarrow 0)$, Eqs. (55), (56), and (57) reduce to their non-deformed versions Eqs. (28), (29), and (30), since $K_{q} \rightarrow p$ and $G^{\prime} \rightarrow G$.

In analogy with the non-deformed case we can write the gap equation in terms of the quark condensates as

$$
M=-2 G^{\prime}\langle\bar{\Psi} \Psi\rangle .
$$

Comparing the two forms of the gap equation Eqs. (42) and (59), we find a new deformed condensate given by

$$
\langle\bar{\Psi} \Psi\rangle=-\frac{N_{c} N_{f}}{\pi^{2}} \int_{0}^{\Lambda} d p p^{2} \frac{M}{\sqrt{\mathbf{K}_{q}^{2}+M^{2}}}
$$


This condensate is different from the one obtained in the previous section, where the condensate was explicitly deformed [4]. It also has exactly the same form of the non-deformed one, but is written in terms of the new variables. It is worth to mention that the new condensate is not obtained by calculating the vacuum expectation value of a deformed scalar density. In fact, it corresponds to the gap equation which arises from the variational procedure started from the $q$-deformed Hamiltonian. The new pion decay constant can also be obtained in analogy to the non-deformed case

$$
F_{\pi}^{2}=N_{c} M^{2} \int_{0}^{\Lambda} \frac{d^{3} p}{(2 \pi)^{3}} \frac{1}{\left(\mathbf{K}_{q}^{2}+M^{2}\right)^{3 / 2}} .
$$

A closer look to the left side of Eq. 55 shows that the dynamical mass has two components: one proportional to the original dynamical mass $m$ and another term which we call $M_{0}$

$$
M=\underbrace{[1+(1-\alpha / 2) Q] \underbrace{p \tan 2 \theta(p)}_{m}}_{M_{d y n}}+\underbrace{p_{0} \tan 2 \theta(p)}_{M_{0}},
$$

where $p_{0}=-\frac{Q G \Lambda^{3}}{\pi^{2}}$ is $P_{0}$ (Eq. 52) for $N_{c}=3$ and $N_{f}=2$. We can then calculate the effect of the deformation on the dynamical mass $m$ :

$$
m=\frac{M}{1+(1-\alpha / 2) Q},
$$

where $M$ is obtained by solving Eq. (59). In an analogous way, the effect on the condensate can be calculated by substituting $M \rightarrow m$ in the numerator of Eq. (60) [15] .

\section{TEMPERATURE AND $q$-DEFORMATION}

In this section we want to study the interplay of temperature and $q$-deformation in the NJL model. We review the standard approach to introduce finite temperature and chemical potential in the NJL model $[2,3]$ in subsections IV.A and IV.B. When temperature is introduced in the model, the condensate is replaced by the thermal expectation value of the scalar density, which contains the Fermi-Dirac distributions. We also have a gap equation for the chemical potential, which is modified by the interactions in such a way that we need to solve a system of coupled gap equations. In subsections IV.C and IV.D, the standard formalism is extended to incorporate the effects of $q$-deformation. In particular, aspects of chiral symmetry restoration and pionic properties in the $q<1$ regime will be discussed.

\section{A. The NJL model at Finite Temperature}

The starting point for the study of the thermodynamics of the NJL model is the partition function

$$
Z=\operatorname{Tr} \exp \left[-\beta\left(\mathcal{H}_{N J L}-\mu_{i} \mathcal{N}_{i}\right)\right],
$$

where $\beta=T^{-1}, \mathcal{N}_{i}$ is the valence quark number operator of flavor $i$, and $\mu_{i}$ are the corresponding chemical potential. From the above partition function, one can calculate the thermal expectation value of an operator $\mathcal{A}$

$$
\langle\langle\mathcal{A}\rangle\rangle=\frac{1}{Z} \operatorname{Tr} \mathcal{A}\left\{\exp \left[-\beta\left(\mathcal{H}_{N J L}-\mu_{i} \mathcal{N}_{i}\right)\right]\right\}
$$

where the operator in question can be, for example, the scalar density $\bar{\psi} \psi$ or the quarks density $\psi^{\dagger} \psi$.

Here the Hamiltonian is given by

$$
\begin{aligned}
\mathcal{H}_{N J L} & =-i \bar{\psi} \gamma \cdot \nabla \psi-G(\bar{\psi} \psi)^{2}-G\left(\bar{\psi} i \gamma_{5} \tau \psi\right)^{2}+m_{0} \bar{\psi} \psi, \\
& =-i \bar{\psi} \gamma \cdot \nabla \psi-\mathcal{L}_{i n t}+m_{0} \bar{\psi} \psi,
\end{aligned}
$$

which, in the mean field approximation, is written as

$$
\mathcal{H}_{M F}=-i \bar{\psi} \gamma \cdot \nabla \psi+m \bar{\psi} \psi+G \sigma_{1}^{2}+\frac{G}{N_{c}} \sigma_{2} \mathcal{N}-\frac{G}{2 N_{c}} \sigma_{2}^{2},
$$

where $m$ is the effective quark mass

$$
m=m_{0}-2 G \sigma_{1},
$$

$\mathcal{N}$ is the number operator

$$
\mathcal{N}=\bar{\psi} \gamma_{0} \psi=\psi^{\dagger} \psi
$$

and $\sigma_{1}, \sigma_{2}$ are defined as

$$
\begin{aligned}
& \sigma_{1}=\langle\langle\bar{\psi} \psi\rangle\rangle, \\
& \sigma_{2}=\left\langle\left\langle\bar{\psi} \gamma_{0} \psi\right\rangle\right\rangle .
\end{aligned}
$$

The Hamiltonian in the mean field approximation represents a system of free particles of mass $m$ and the chemical potential is given by

$$
\mu=\mu_{0}-\frac{G}{N_{c}} \sigma_{2}
$$

where $\mu_{0}$ is the chemical potential when there is no vector interaction, generated by the Fierz transformation of $\mathcal{L}_{\text {int }}$. Equations (69) and (73) form a system of self-consistent coupled equations

$$
\left\{\begin{array}{l}
m=m_{0}-2 G \sigma_{1} \\
\mu=\mu_{0}-\frac{G}{N_{c}} \sigma_{2}
\end{array}\right.
$$

Solving the above equations we obtain the effective mass and chemical potential, which we can put back in the expressions for $\sigma_{1}$ and $\sigma_{2}$ to obtain the condensate and the density at a given temperature $T$.

\section{B. Thermal Expectation Values}

The dynamical mass calculation depends on expectation values containing the Fermi-Dirac distributions. The thermal expectation values relevant for the NJL model, namely $\sigma_{1}$ and $\sigma_{2}$, can be calculated within the formalism of the thermal Green function [11], which has the advantage of separating the parts that depend on the temperature and the chemical potential. The thermal Green function for a free fermion at a temperature $T$ and chemical potential $\mu$ is written as 

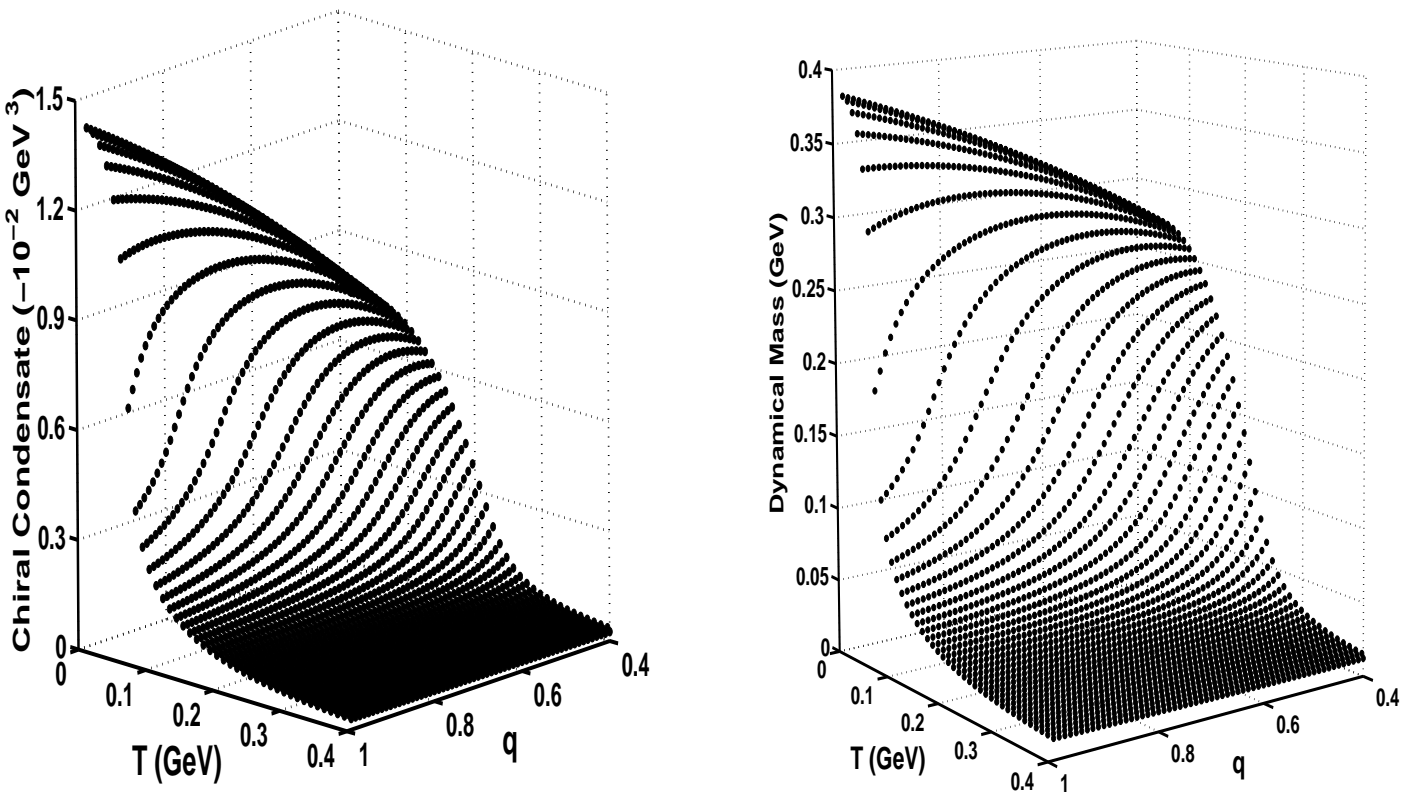

FIG. 1: Chiral condensate (in units of $-10^{-2} \mathrm{GeV}^{3}$ ) and dynamical quark mass as functions of both temperature and $q$-deformation in the $q<1$ regime. The results presented in the figures were obtained with the following parameters: $G=6.58 \mathrm{GeV}^{-2}, \Lambda=0.6 \mathrm{GeV}, m_{0}=5.87$ $\mathrm{MeV}$, and $\mu_{0}=0.35 \mathrm{GeV}$.

$$
\begin{aligned}
S_{F}(p ; T, \mu) & =\frac{\not p+m}{p^{2}-m^{2}+i \varepsilon}+2 \pi i \delta\left(p^{2}-m^{2}\right) \\
& \times(\not p+m)\left[\theta\left(p^{o}\right) n(\mathbf{p}, T, \mu)+\theta\left(-p^{o}\right) \bar{n}(\mathbf{p}, T, \mu)\right],
\end{aligned}
$$

where

$$
n(\mathbf{p}, T, \mu)=\frac{1}{1+\exp [-\beta(E-\mu)]},
$$

and

$$
\bar{n}(\mathbf{p}, T, \mu)=\frac{1}{1+\exp [-\beta(E+\mu)]},
$$

are the fermions and anti-fermions distribution function respectively with $E=\sqrt{\mathbf{p}^{2}+m^{2}}$.

Making use of the anti-commutation relation for the fermionic fields, the expectation values can be written as

$$
\begin{aligned}
\langle\langle\bar{\psi}(x) \psi(x)\rangle\rangle & =-\operatorname{Tr} \lim _{y \rightarrow x+}\langle\langle T[\psi(x) \bar{\psi}(y)]\rangle\rangle, \\
\left\langle\left\langle\bar{\psi} \gamma_{0} \psi\right\rangle\right\rangle & =-\operatorname{Tr} \lim _{y \rightarrow x+} \gamma_{0}\langle\langle T[\psi(x) \bar{\psi}(y)]\rangle\rangle .
\end{aligned}
$$

The term on the right-hand side of the above equations is the definition of the thermal Green function in the configuration space:

$$
i S_{F}(x-y ; T, \mu)=\langle\langle T[\psi(x) \bar{\psi}(y)]\rangle\rangle .
$$

The expectation values are then re-written as

$$
\begin{aligned}
\langle\langle\bar{\psi}(x) \psi(x)\rangle\rangle & =-i \lim _{y \rightarrow x+} \operatorname{Tr} \int \frac{d^{4} p}{(2 \pi)^{4}} S_{F}(p ; T, \mu) e^{-i(x-y) p} \\
\left\langle\left\langle\bar{\psi} \gamma_{0} \psi\right\rangle\right\rangle & =-i \lim _{y \rightarrow x+} \operatorname{Tr} \int \frac{d^{4} p}{(2 \pi)^{4}} \gamma_{0} S_{F}(p ; T, \mu) e^{-i(x-y) p}
\end{aligned}
$$

Applying a cutoff in the momentum, the results for $\sigma_{1}$ and $\sigma_{2}$ are the following

$$
\begin{aligned}
& \sigma_{1}=\langle\langle\bar{\psi} \psi\rangle\rangle=-\frac{N_{c} N_{f}}{\pi^{2}} \int_{0}^{\Lambda} d p p^{2} \frac{m}{E}[1-n-\bar{n}], \\
& \sigma_{2}=\left\langle\left\langle\bar{\psi} \gamma_{0} \psi\right\rangle\right\rangle=\frac{N_{c} N_{f}}{\pi^{2}} \int_{0}^{\Lambda} d p p^{2}[n-\bar{n}],
\end{aligned}
$$

and the barion density can be written as

$$
\rho_{B}=\frac{1}{3}\left\langle\left\langle\bar{\psi} \gamma_{0} \psi\right\rangle\right\rangle=\frac{N_{c} N_{f}}{3 \pi^{2}} \int_{0}^{\Lambda} d p p^{2}[n-\bar{n}]
$$




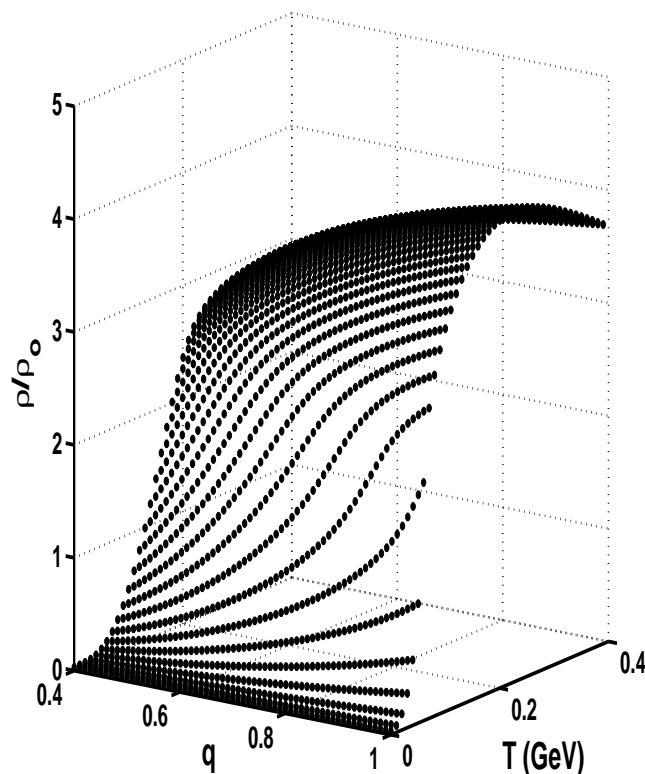

FIG. 2: The barion density in units of the nuclear matter density $\rho_{0}=0.15 \mathrm{fm}^{-3}$ as a function of both temperature and $q$-deformation in the $q<1$ regime.

\section{Condensates, mass, and chiral symmetry restoration}

An interesting window to the application of $q$-deformation to hadronic physics can be opened for $q$-values smaller than one. It is tempting to explore the behavior of the condensate in this new regime for smaller values of $q$, even considering that the truncation at order $Q$ may not be granted. In this case, we observed in [5] that the chiral symmetry is restored in the limit $q \rightarrow 0$, since the condensate vanishes. It is then worth to investigate the effect of both temperature and $q$-deformation in the chiral symmetry restoration process as well as in the pion properties.

In order to study the effect of both temperature and $q$ deformation in the chiral symmetry restoration process, we can replace the gap equation for the mass in the system of coupled equations by its deformed version Eq. (86)

$$
M=m_{0}-2 G^{\prime}\langle\langle\bar{\Psi} \Psi\rangle\rangle,
$$

so that the new system of coupled equation we need to solve is

$$
\left\{\begin{array}{l}
M=m_{0}-2 G^{\prime} \Sigma_{1}, \\
\mu=\mu_{0}-\frac{G}{N_{c}} \sigma_{2},
\end{array}\right.
$$

where $\Sigma_{1}$ represents the deformed version of the thermal condensate $\sigma_{1}$.

Solving the new set of coupled gap equations we can observe the condensate as a function of temperature for different values of the deformation parameter. It is important to mention that the effects of the algebra deformation come from gap equation for the mass. However, the chemical potential is also affected once it depends on the mass through the coupled equations. Thus, for a given temperature, we obtain the mass and the chemical potential by solving the system of coupled gap equations. We then calculate the condensate and the density.

In order to visualize the chiral symmetry restoration process with the influence from both temperature and deformation, we plot the surfaces shown in Fig. (1) where the chiral condensate and the constituent quark mass are plotted as functions of both temperature and $q$-deformation.

At $q<1$ deformations, an interesting result is observed: the chiral symmetry restoration is similar to the one observed by Klimt et al. as function of temperature and density [13]. However, the process is slower as $q$ gets smaller when compared with the case of $\rho$ getting larger. Figure 1 shows that the condensate is substantially affected by the deformation and lowers as $q$ decreases, while the effect of temperature is softened. The same behavior is observed in the dynamical quark mass since it is linearly constrained to the condensate by the gap equation.

We have also calculated the barion density as a function of $T$ and $q$, which is displayed in Fig. 2. The effect of the deformation on the barion density in only observed as the temperature increases. The density also lowers as $q$ decreases, and the temperature effect is also smoothed down.

The behavior of the dynamical mass and the chiral condensate directs to chiral symmetry restoration. We would therefore expect the pion to become massless in the limit $q \rightarrow 0$. In the next section, we evaluate the pion decay constant and mass in order to investigate the pion properties in a regime where condensate is lower than in the standard NJL model due to the effects of the $q$-deformation.

\section{Pion Properties in the $q<1$ regime}

Recent works [12] have shown that the spontaneous chiral symmetry breaking may result from a balance of the density of states and their mobility in the medium. It may occur with a vanishing $\langle\bar{q} q>$ condensate as long as the low density of states is compensated by a high mobility. In this case, the condensate is no longer the order parameter.

The effective models for QCD, like the NJL, fail to describe hadronic observables when the condensate is small or null. The $q$-deformed version of the NJL model seems to be an alternative to study the low condensation scenario. As a first application, we start with the pion mass since it cannot be reproduced in the standard NJL model in a low condensation regime.

With a fixed value for the current quark mass, after obtaining the pion decay constant through Eq. 61, we obtain the pion mass by using the Gell-Mann-Oakes-Renner (GOR) relation

$$
m_{\pi}^{2} F_{\pi}^{2}=\left(m_{0}^{u}+m_{0}^{d}\right) \times|\langle\bar{\Psi} \Psi\rangle|,
$$

written in terms of our deformed quantities. The pion properties (decay constant and mass) as functions of both temperature and $q$-deformation are shown in Figure 3. In order to 

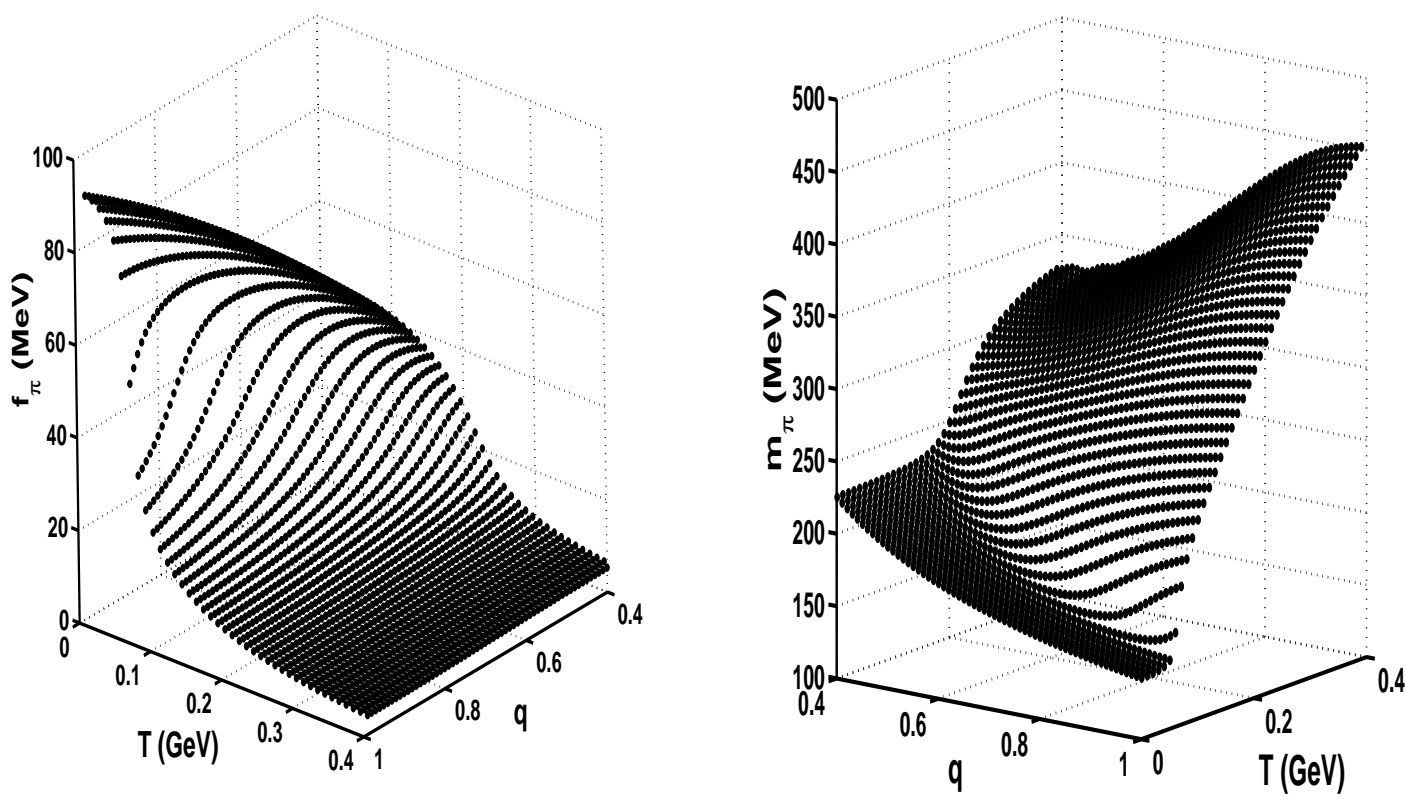

FIG. 3: The pion decay constant and the pion mass as functions of both temperature and $q$-deformation in the $q<1$ regime.

have an idea of the effect of the deformation in a low condensation regime, we consider the case of $q=0.5$, where condensation is approximately $35 \%$ lower than in the non-deformed case (see Fig. 1). In this particular scenario, we obtain a pion mass of about $190 \mathrm{MeV}$. The standard NJL model would give a pion mass of about $100 \mathrm{MeV}$ in such condensation scenario. The correlations between the constituents of the system introduced when the underlying $s u(2)$ algebra is deformed seems to compensate the low condensation, which is responsible for keeping a reasonable mass for the pion even when the value of the condensate is getting smaller.

\section{FINAL REMARKS}

So far we have performed the $q$-deformation of the NJL model with finite temperature also taken into account. We studied the $q$-deformation of the underlying $s u(2)$ algebra in a two flavor version of the NJL model and investigated an important feature of chiral symmetry: its restoration at finite temperature. Also, we studied the pion properties in a low condensation scenario, where the standard NJL model would underestimate the pion mass.

As far as the effects of both temperature and deformation are concerned, our main conclusions can be summarized as follows. The quark condensate and the dynamical mass decrease as $q$ gets smaller than one, and chiral symmetry would be restored in the limit $q \rightarrow 0$ [5]. This effect is specially observed at low temperatures, since we have thermal restoration at large $T$. Surprisingly, as the condensate lowers due to the deformation, the pion not only keeps massive but also increases. The GOR relation, Eq. 88, with our deformed quantities gives the reason for this behavior, where the pion decay constant decreases faster than the square root of the condensate.

It seems that the effect of the deformation compensates the lower condensation, suggesting that the quarks' mobility is affected when the algebra is deformed. In fact, the correlations introduced by the deformation generate an extra momentum $P_{0}$ for the constituents of the system (see Eq. 52) which is responsible for enhancing the mobility in the medium.

\section{Acknowledgments}

The authors are grateful to U-G. Meißner and M. R. Robilotta for the suggestion which motivated our study on the $m_{\pi}$ and $f_{\pi}$ behavior, and to D. Galetti and B. M. Pimentel for very helpful discussions. This work was supported by FAPESP grant number 2002/10896-7. V. S. T. would like to thank FAEPEX/UNICAMP for financial support.
[1] Y. Nambu and G. Jona-Lasinio, Physical Review 122, 345 (1961).

[2] M. Asakawa and K. Yasaki, Nucl. Phys. A504, 668 (1989).
[3] U. Vogl and W. Weise, Prog. Part. Nucl. Phys. 27, 195 (1991), and references therein.

[4] V. S. Timóteo and C. L. Lima, Phys. Lett. B 448, 1 (1999). 
[5] V. S. Timóteo and C. L. Lima, Mod. Phys. Lett. A15, 219 (2000).

[6] S. P. Klevansky, Reviews of Modern Physics 64, 649 (1992).

[7] D. Galetti and B. M. Pimentel, An. Acad. Bras. Ci. 67, 7 (1995); S. S. Avancini, A. Eiras, D. Galetti, B. M. Pimentel, and C. L. Lima, J. Phys. A: Math. Gen. 28, 4915 (1995); D. Galetti, J. T. Lunardi, B. M. Pimentel, and C. L. Lima, Physica A242, 501 (1997).

[8] M. Ubriaco, Phys. Lett. A 219, 205 (1996)

[9] L. Tripodi and C. L. Lima, Phys. Lett. B 412, 7 (1997).

[10] J. Gasser and H. Leutwyler, Ann. Phys. 158, 142 (1984).
[11] L. Dolan and R. Jackiw, Phys. Rev. 9, 3320 (1974).

[12] J. Stern, hep-ph/9801282.

[13] S. Klimt, M. Lutz, and W. Weise, Phys. Lett. B 249, 386 (1990).

[14] Figure 1 of Ref. [5] shows the variational angle as a function of $q$ when the dynamical mass is equal to $p$, not $300 \mathrm{MeV}$ as stated in the caption.

[15] The term $M_{0}$ has to be considered only when solving the gap equation. But it can be neglected when going from Eq. (62) to (63), since it is much smaller than the dynamical mass, $M$. 